\title{
A novel endogenous damage signal, glycyl tRNA synthetase, activates multiple beneficial functions of mesenchymal stem cells
}

\author{
Se-Ra Park ${ }^{1,2} \cdot$ Hyun-jin Kim ${ }^{1,2} \cdot$ Se-Ran Yang ${ }^{3} \cdot$ Chan Hum Park ${ }^{4} \cdot$ Hwa-Yong Lee ${ }^{5}$ In-Sun Hong ${ }^{1,2}$
}

Received: 15 August 2017 / Revised: 6 February 2018 / Accepted: 6 March 2018 / Published online: 17 April 2018

(c) ADMC Associazione Differenziamento e Morte Cellulare 2018

\begin{abstract}
During tissue repair, the injury site releases various bioactive molecules as damage signals to actively recruit stem cells to the damaged region. Despite convincing evidence that mesenchymal stem cells (MSCs) can sense damage signals and promote repair processes, the identity of these signals and how these signals regulate stem cell-mediated tissue repair remain unknown. Glycyl tRNA synthetase (GRS) is a ubiquitously expressed enzyme that catalyzes the first step of protein synthesis in all organisms. In addition to this canonical function, we identified for the first time that GRS is released by damaged tissues or cells in response to various injury signals and may function as a damage signal that activates the proliferative, differentiation, and migratory potential of MSCs, possibly through its identified receptor, cadherin-6 (CDH-6). Binding between GRS and CDH-6 activates survival signals, such as those of the PI3K/Akt and/or FAK/ERK1/2 pathways. More importantly, we also found that MSCs stimulated with GRS show significantly improved homing and differentiation potential and subsequent in vivo therapeutic effects, in a liver fibrosis animal model. Collectively, our findings provide compelling evidence for a novel function of GRS in enhancing the multiple beneficial functions of stem cells via a noncanonical mechanism as a damage signal.
\end{abstract}

Electronic supplementary material The online version of this article (https://doi.org/10.1038/s41418-018-0099-2) contains supplementary material, which is available to authorized users.

Hwa-Yong Lee

hylee@jwu.ac.kr

$\triangle$ In-Sun Hong

hongstem@gachon.ac.kr

1 Laboratory of Stem Cell Research, Lee Gil Ya Cancer and Diabetes Institute, Gachon University, Incheon 406-840, Republic of Korea

2 Department of Molecular Medicine, School of Medicine, Gachon University, Incheon 406-840, Republic of Korea

3 Department of Thoracic and Cardiovascular Surgery, Kangwon National University, Chuncheon, South Korea

4 Department of Otolaryngology-Head and Neck Surgery, Chuncheon Sacred Heart Hospital, Hallym University College of Medicine, Chuncheon, South Korea

5 Department of Biomedical Science, Jungwon University, 85 Goesan-eup, Munmu-ro, Goesan-gun 367-700, Republic of Korea

\section{Introduction}

Aminoacyl tRNA synthetases (AARSs) form a group of ubiquitously expressed enzymes that catalyze the first step of protein synthesis in all organisms, as they attach a specific amino acid to their cognate tRNAs to form an aminoacyl tRNA [1]. Therefore, until recently, AARSs have been considered homeostatic or housekeeping enzymes. Interestingly, in addition to this canonical function, several recent studies have suggested that some AARS family members may have additional cytokine-like activities in multiple physiological conditions, such as glucose homeostasis [2], inflammation [3], angiogenesis [4], cell proliferation [5], and apoptosis [6]. Most recently, particular attention has been devoted to the non-canonical functions of the AARS family member glycyl tRNA synthetase (GRS), in nerve disease and damage [7-9], as it was selectively secreted in specific disease conditions and regulated the outcome of these diseases. However, the non-canonical functions and molecular mechanisms of GRS remain illdefined.

Mesenchymal stem cells (MSCs) have shown significant therapeutic potential for tissue regeneration because of their ability to stimulate angiogenesis and migration and to 
promote the differentiation and growth of local progenitor or stem cells $[10,11]$. More importantly, MSCs are recruited to damaged or diseased sites in response to various danger signals and subsequently promote tissue regeneration [12-14]. However, the mechanisms by which MSCs are recruited to the sites of tissue damage and mediate multiple beneficial effects remain unclear. We hypothesized that GRS may actively be released from damaged tissue as a danger signal and subsequently promote tissue regeneration by stimulating multiple beneficial functions of MSCs. Indeed, we showed for the first time that GRS is actively secreted by multiple human cell types in response to various injury signals both in vitro and in vivo and then acts as a potent stimulatory factor that facilitates MSCs proliferation differentiation, and migration. Furthermore, we found that downregulation of cadherin-6 (CDH-6) significantly attenuates the GRS-mediated beneficial functions of MSCs, suggesting that $\mathrm{CDH}-6$ is a functional receptor for GRS. We subsequently explored the molecular mechanism underlying the stimulatory effects of GRS on multiple MSCs functions. Interestingly, GRS activates survival pathways, such as the PI3K/Akt and FAK/ERK1/2 signaling cascades, which are involved in various physiological functions, including cell proliferation $[15,16]$, differentiation $[15,17,18]$, and migration $[15,19]$. Consistently, inhibition of these signaling pathways with specific inhibitors significantly attenuates the GRS-induced stimulatory effects on MSCs. These results indicate that GRS stimulates MSCs growth, differentiation, and homing via PI3K/Akt and/or FAK/ ERK1/2 signaling. Another key finding from our study is that the in vivo therapeutic effects of MSCs can be significantly enhanced upon stimulation with GRS in a liver fibrosis animal model. Taken together, these findings suggest that in addition to its previously reported canonical activities, GRS is actively secreted in response to tissue damage as an endogenous danger signal and subsequently enhances the therapeutic effects of MSCs by increasing multiple beneficial functions via PI3K/Akt and/or FAK/ ERK1/2 signaling.

\section{Results}

\section{GRS is actively secreted in response to tissue damage in vitro and in vivo}

We first isolated MSCs from human adipose tissue (Suppl. Figure 1A) and then characterized their biological properties by using multiple negative and positive MSC surface markers (Suppl. Figure 1B). Their capacity to differentiate into multiple lineages was evaluated by inducing adipogenic and osteogenic differentiation (Suppl. Figure 1C). To investigate whether GRS is secreted from damaged or stressed cells in response to injury, multiple cell types, including fibroblasts, MSCs, and vascular endothelial cells (ECs), were exposed to various injury conditions, such as oxidative stress, radiation damage, and starvation. GRS secreted into the culture media was precipitated using $10 \%$ trichloroacetic acid (TCA), as previously described [20]. As shown in Fig. 1a-d, various types of cells actively secreted GRS into the culture media in response to cellular damage or stress in vitro. Additionally, to determine entry points of GRS secretion after injuries, these cells were exposed to various injury conditions, and then secreted proteins were sampled at many time points. While it appears to vary depending on what types of cells or injury conditions, injured cells usually secreted GRS into the culture media in response to damage after $24 \mathrm{~h}$. The observed GRS secretion kinetics showed a clearly time-dependent manner with a peak release $72 \mathrm{~h}$ after injury (Supple Fig. 2A-C). We have also observed basal in vitro secretions of GRS from various cell types without damage induction, suggesting that the basal secretion of GRS exist among various cell types under normal circumstances (Supple Fig. 2A-C). To further verify in vivo whether tissue damage can stimulate GRS secretion into the blood circulation, systemic GRS levels in peripheral blood samples from mice with carbon tetrachloride $\left(\mathrm{CCl}_{4}\right)$ induced hepatic damage were examined. Apart from hepatic fibrosis and enlarged spleens (Suppl. Figure 3A), histological examination revealed that hepatocytes of the liverinjured mice exhibited high centrilobular necrosis (Suppl. Figure 3B). Consistent with this result, the levels of various liver injury markers, such as alanine amino transferase (ALT), aspartate amino transferase (AST), and total bilirubin, were significantly higher in the liver-injured mice than in the control mice (Suppl. Figure 3C). The acute liver damage resulted in a significant increase in GRS secretion into the peripheral circulation compared with that in the uninjured control mice (Fig. 1e). We further confirmed the tissue damage-induced GRS levels in the peripheral circulation using ELISA (Fig. 1f). These results suggest that GRS is actively secreted from multiple cell types in response to various injury signals both in vitro and in vivo as an endogenous danger signal.

\section{GRS enhances multiple beneficial functions of MSCs in vitro}

As GRS might play a role as a systemic danger signal of tissue injury, we investigated whether GRS enhances the various beneficial functions of MSCs in vitro. We first examined the proliferative response of MSCs to GRS exposure. We found steadily increasing growth rates in MSCs treated with GRS compared with the non-treated control cells (Fig. 2a). More strikingly, GRS significantly increased MSC migration (Fig. 2b). To further confirm the 
A

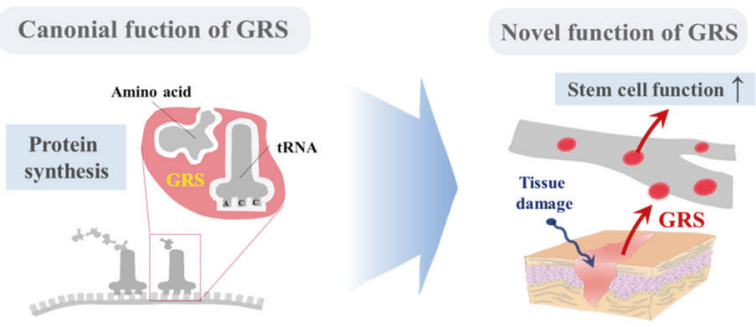

C

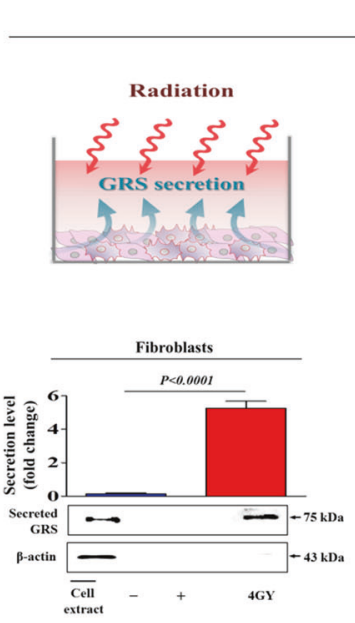

Radiation

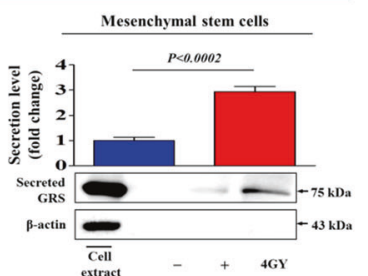

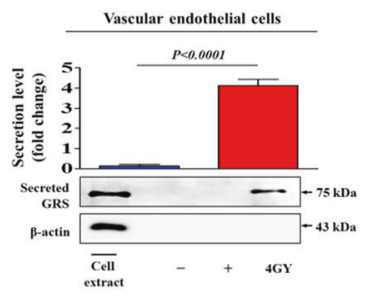

B

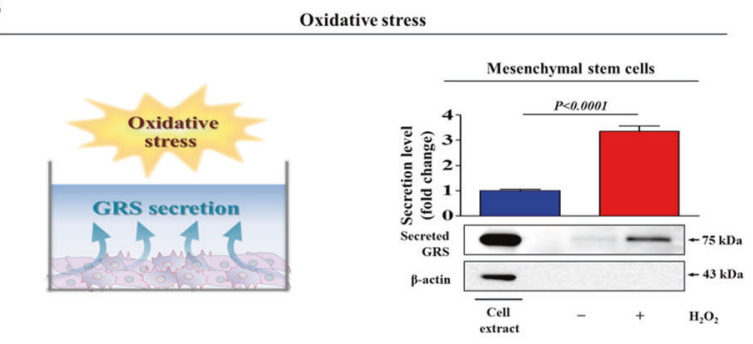

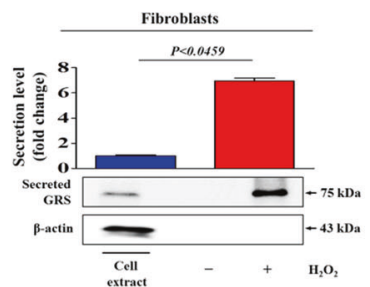

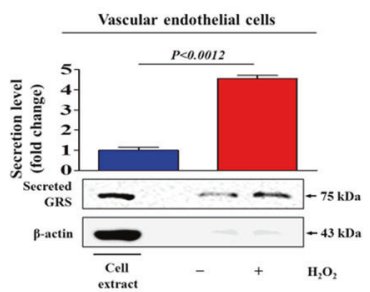

D Serum depletion
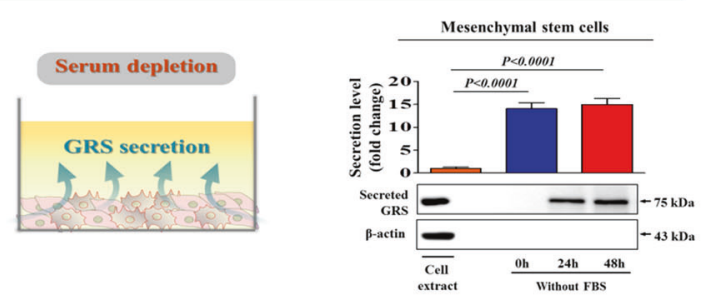

$\mathbf{E}$

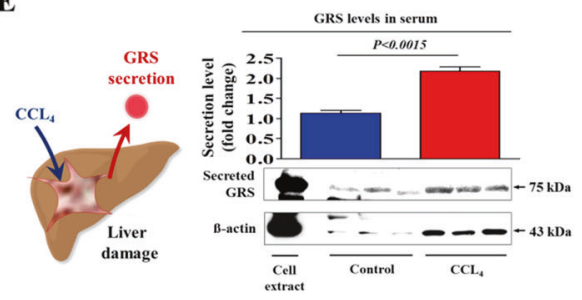

F

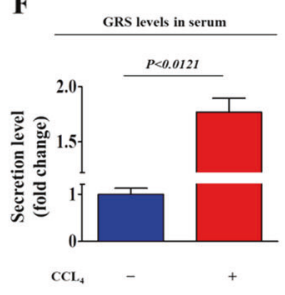

Fig. 1 GRS is actively secreted by multiple cell types in response to various injury signals. GRS has been considered a housekeeping enzyme that catalyzes the first step of protein synthesis. In addition to this canonical function, GRS is selectively secreted under specific damage conditions (a). Fibroblasts, MSCs, and vascular ECs were incubated in standard culture medium with or without $\mathrm{H}_{2} \mathrm{O}_{2}(10 \mathrm{mM})$ for $30 \mathrm{~min}$, after which the medium was replaced with serum-free medium and the cells were cultured for $48 \mathrm{~h}$ (b). Fibroblasts, MSCs, and vascular ECs were exposed to acute irradiation at a dose of $4 \mathrm{~Gy}$ (X-ray), after which the medium was replaced with serum-free medium and the cells were cultured for $48 \mathrm{~h}(\mathbf{c})$. Fibroblasts, MSCs, and vascular ECs were cultured with or without serum for $24 \mathrm{~h}$ and $48 \mathrm{~h}$ (d). Proteins in the TCA-precipitated supernatants were analyzed by western blotting using an anti-human GRS polyclonal antibody $(\alpha-$ GRS). Acute $\mathrm{CCl}_{4}$ treatment $(800 \mathrm{mg} / \mathrm{kg}$, administered intraperitoneally) produced significant histological liver damage. After albumin/immunoglobulin depletion, the proteins in the serum samples were precipitated with $10 \%$ TCA and subjected to SDS-PAGE. $\mathrm{CCl}_{4}$ induced acute liver failure resulted in a significant increase in GRS secretion into the peripheral circulation compared with that of the uninjured control mice (e). Increased GRS concentrations by $\mathrm{CCl}_{4}$ treatment in the serum samples were detected by ELISA (f). $\beta$-actin was used as the internal control. The results represent the mean \pm SD from three independent experiments stimulatory effect of GRS on the migratory ability of MSCs, western blotting was used to evaluate the expression levels of matrix metalloproteinase 2 (MMP-2) and MMP-9, which play important roles in regulating cell migration (Fig. 2c). Previous studies have indicated that the actin cytoskeleton is involved in cell migration via pushing or pulling on the substrate near the plasma membrane [21]. Consistently, phalloidin staining of actin filaments revealed a strong correlation between the GRS treatment and high actin cytoskeleton disorganization (Fig. 2d), suggesting that the markedly increased migration capability of GRS-treated MSCs may be related to actin cytoskeleton disorganization. GRS also significantly increased the proliferation (Suppl. Figure 4A) and migration (Suppl. Figure 4B and C) potential of other cell types, such as fibroblasts and vascular ECs. Importantly, GRS significantly increased multi-lineage differentiation potential toward osteoblasts and adipocytes in vitro (Fig. 2e) without affecting the levels of MSC- 
A

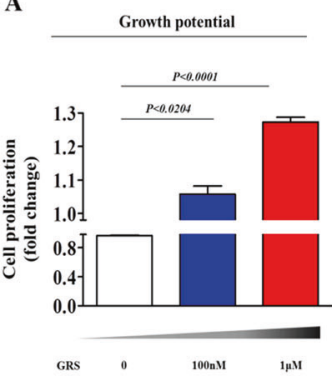

B

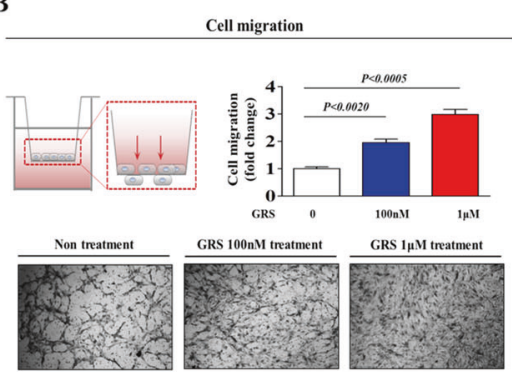

C

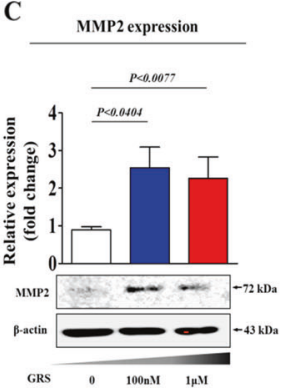

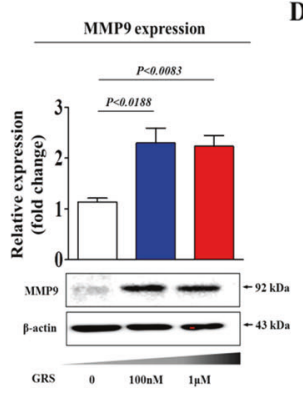

D

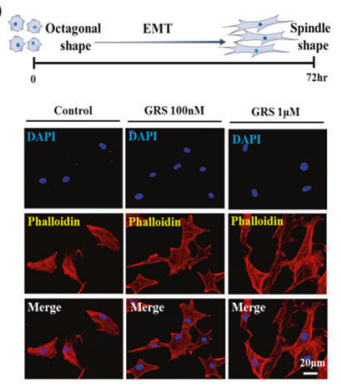

$\mathbf{E}$
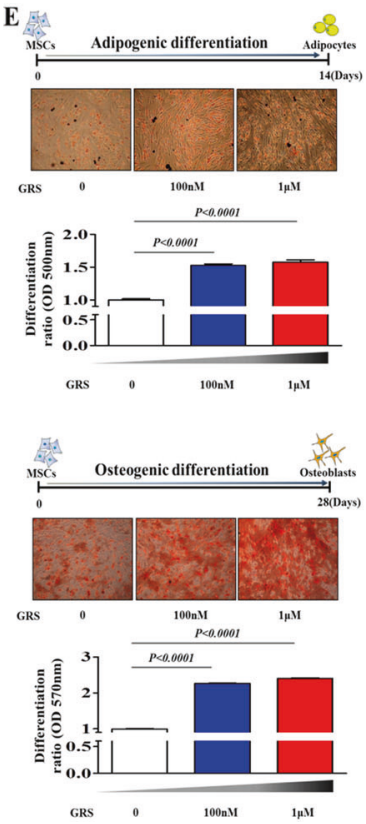

$\mathbf{F}$
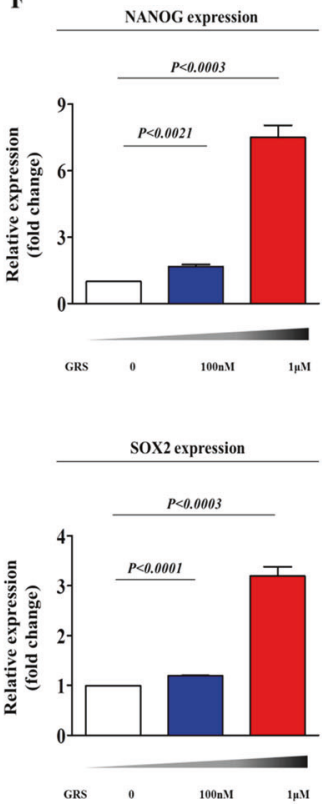

Fig. 2 GRS promotes multiple beneficial MSC functions in vitro. The stimulation of MSC viability by GRS treatment for $72 \mathrm{~h}$ was determined by an MTT assay. MSC viability (\%) was calculated as a percent of the vehicle control (a). MSCs were treated with GRS for 24 $\mathrm{h}$, after which the effect of GRS on MSC migration ability was evaluated using the transwell migration assay. GRS treatment significantly increased MSC migration across the membrane compared with the negative control (b). The relative expression levels of key positive regulators of cell migration (MMP-2/9) were assessed using western blotting (c). GRS-induced actin filament disorganization and the morphological transition of MSCs were visualized by staining the actin filaments with phalloidin (d). Schematic representation of the experimental protocol as described in the materials and methods section. Confluent MSCs were cultured in osteogenic or adipogenic medium with or without GRS. The effects of GRS on osteoblast and adipocyte differentiation were determined by alizarin red and oil red $\mathrm{O}$ staining, respectively. The relative quantification of calcium mineral content and lipid droplet formation was determined by measuring absorbance at $570 \mathrm{~nm}$ and $500 \mathrm{~nm}$, respectively (e). Real-time PCR results demonstrating changes in the expression of the stem cell markers NANOG and SOX2 after GRS treatment for $24 \mathrm{~h}(\mathbf{f})$. DAPI staining was used to label the nuclei. $\beta$-actin was used as the internal control. The data represent the mean \pm SD from three independent experiments specific positive (CD44, CD73, and CD105) and negative (CD34 and CD45) markers (Suppl. Figure 5). Consistently, the expression levels of the pluripotency-associated factors NANOG and SOX2 were significantly increased by GRS treatment (Fig. 2f). Taken together, these results suggest that GRS promotes multiple beneficial functions, such as the proliferative, migratory, and multi-lineage differentiation potential of MSCs in vitro.

\section{GRS stimulates multiple MSC functions through its functional receptor $\mathrm{CDH}-6$}

Recent studies have suggested that another secreted AARS family member, tryptophanyl tRNA synthetase (TrpRS), binds to VE-cadherin to inhibit angiogenesis [22] and that GRS exerts its anti-tumorigenic activity by binding to K-cadherin (CDH-6) among a set of tumor cell types [20]. Therefore, we analyzed whether GRS can bind to CDH-6 in multiple cell types, including fibroblasts, MSCs, and vascular ECs. Importantly, our results showed that GRS treatment significantly increased $\mathrm{CDH}-6$ expression in all three cell types examined (Fig. 3a). Immunoprecipitation with antibodies directly targeting CDH-6 revealed that GRS could directly bind to CDH-6 in these cells (Fig. 3b). Additionally, to further confirm the basal and damageinduced coupling between GRS and CDH-6, we performed the additional set of immunoprecipitation with $\mathrm{CDH}-6$ antibody under various injury conditions without GRS treatment in multiple cell types. As expected, our immunoprecipitation results revealed that basal and damageinduced GRS could directly bind to $\mathrm{CDH}-6$ in multiple cell types, including fibroblasts, MSCs, and vascular endothelial cells (Supple Fig. 6A-C). Next, to further investigate whether CDH-6 can act as a functional receptor for GRS in MSCs, stable CDH-6 knockdown was obtained by transfection with shRNA targeting CDH-6 (Suppl. Figure 7). Importantly, GRS-induced proliferative (Fig. 3c) and migration abilities (Fig. 3d, e) were significantly attenuated by $\mathrm{CDH}-6$ depletion. In addition, we found that GRSinduced adipogenic and osteogenic differentiation was markedly disrupted by CDH-6 knockdown (Fig. 3f). These results suggest that $\mathrm{CDH}-6$ may serve as a functional receptor to regulate the multiple beneficial MSC functions mediated by GRS. 
A

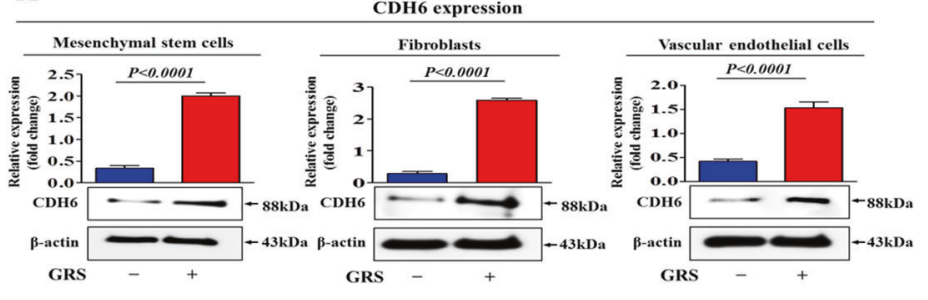

C

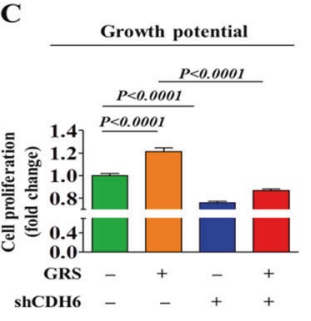

D

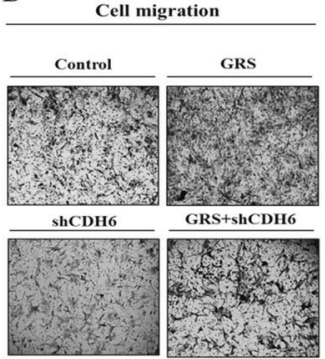

B Direct interation between CDH6 and GRS
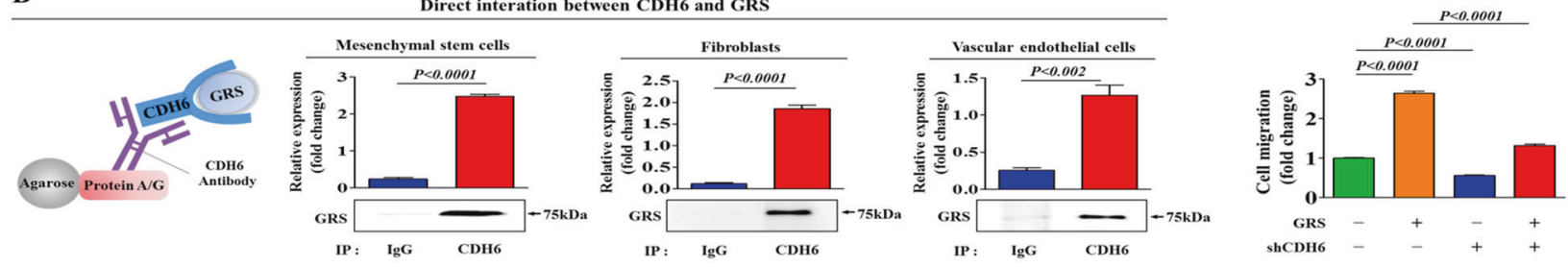

E MMP2 expression

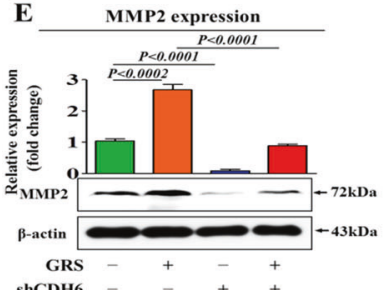

F
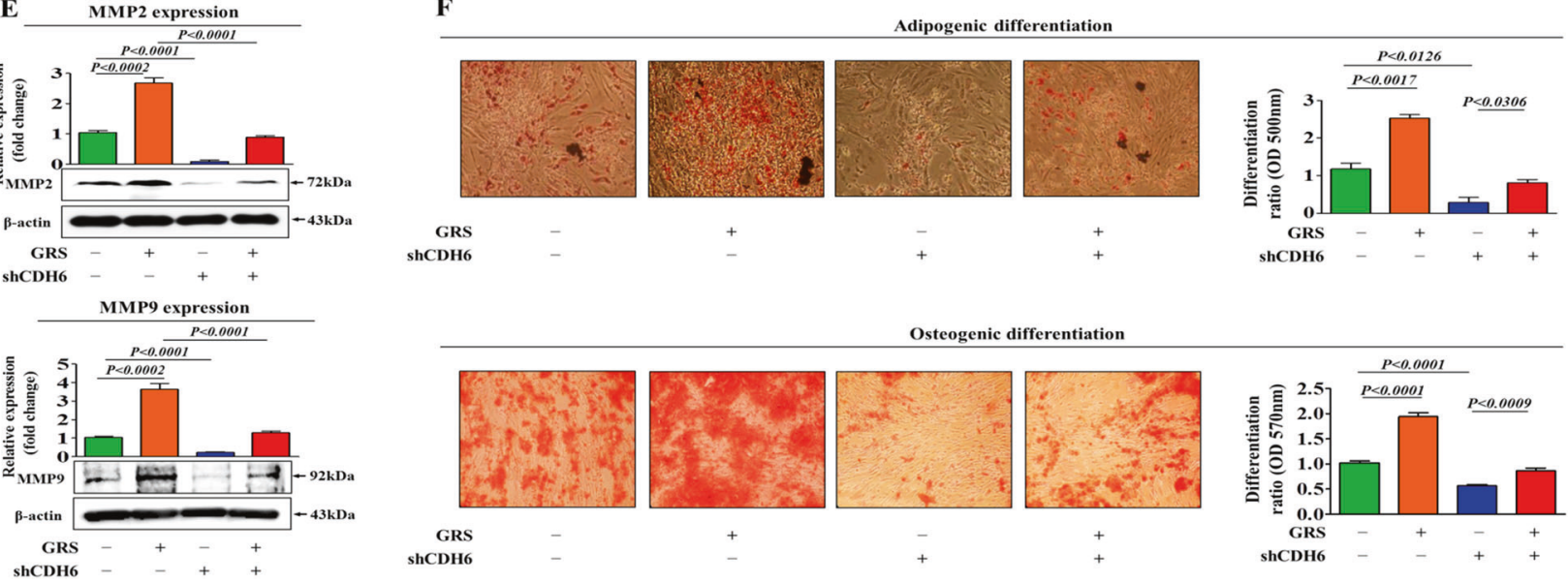

Fig. 3 GRS stimulates multiple beneficial MSC functions through CDH-6. Fibroblasts, MSCs, and vascular ECs were treated with or without GRS $(1 \mu \mathrm{M})$ for $48 \mathrm{~h}$, after which $\mathrm{CDH}-6$ expression was confirmed by western blotting (a). Fibroblasts, MSCs, and vascular ECs were treated with or without GRS $(1 \mu \mathrm{M})$ for $48 \mathrm{~h}$, after which the cell lysates were immunoprecipitated with polyclonal antibodies against CDH-6. We detected GRS on the same membrane by western blot. This result implies the co-precipitation of GRS and CDH-6 (b). MSCs were treated with $1 \mu \mathrm{M}$ GRS alone or were concomitantly transfected with shRNA targeting $\mathrm{CDH}-6$; subsequent changes in cell

viability were measured by an MTT assay (c). Changes in the migratory capacity were measured by the transwell assay (d) and western blotting for MMP-2 and MMP-9 (e). The inhibitory effect of CDH-6 knockdown on GRS-induced osteoblast and adipocyte differentiation was determined by alizarin red and oil red $\mathrm{O}$ staining, respectively. The relative quantification of calcium mineral content and lipid droplet formation was determined by measuring absorbance at $570 \mathrm{~nm}$ and $500 \mathrm{~nm}$, respectively (f). $\beta$-actin was used as the internal control. The data represent the mean $\pm \mathrm{SD}$ from three independent experiments

\section{PI3K/Akt and/or FAK/ERK1/2 signaling mediates beneficial GRS-induced MSC functions}

To investigate the underlying molecular mechanisms of the beneficial GRS-induced effect, we examined the effect of GRS on PI3K/Akt and/or FAK/ERK1/2 signaling, which has been associated with the maintenance [23], differentiation [24], and migration [25] of stem cells. Using western blot analysis, we evaluated whether the GRS treatment was sufficient to activate the PI3K/Akt and/or FAK/ERK1/2 signaling cascade in MSCs. Importantly, the phosphorylation levels of these signaling molecules were significantly increased in GRS-treated cells (Fig. 4a, b). We then examined the effect of CDH-6 knockdown on GRSinduced PI3K/Akt and/or FAK/ERK1/2 signaling. As expected, GRS-induced PI3K/Akt and/or FAK/ERK1/ 2 signaling was significantly attenuated by $\mathrm{CDH}-6$ depletion (Fig. 4c, d). Next, to determine whether the blockade of these signaling activities attenuates the multiple beneficial MSC functions mediated by GRS, we evaluated the effects of the ERK1/2 inhibitor PD98059 and the Akt inhibitor V on MSC growth, migration and differentiation with or without GRS treatment. Indeed, the GRS-induced growth and expression of the proliferation marker Ki67 were also 
A

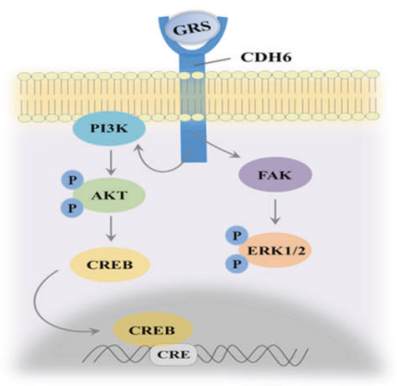

PI3K phosphorylation levels

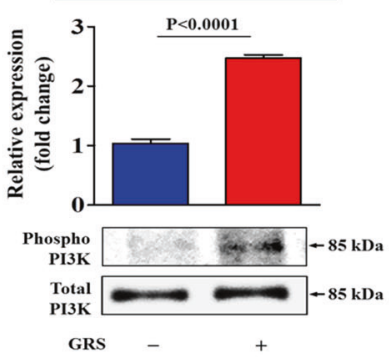

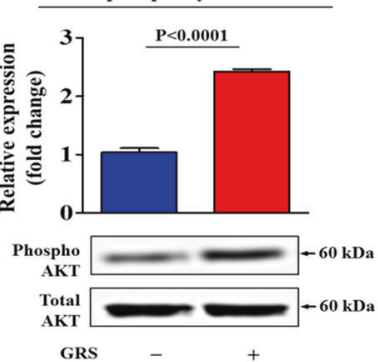

CREB phosphorylation levels

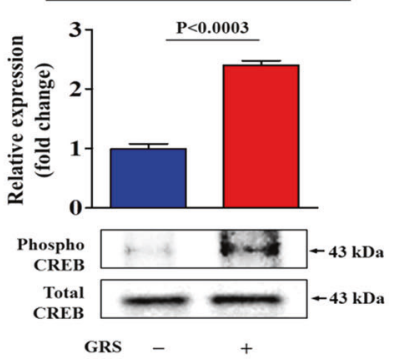

B

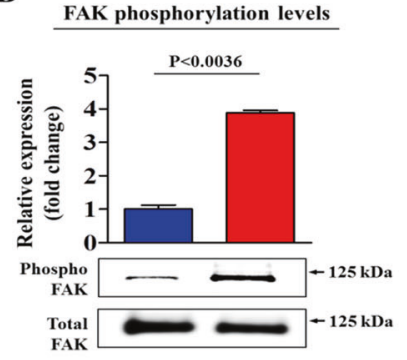

GRS

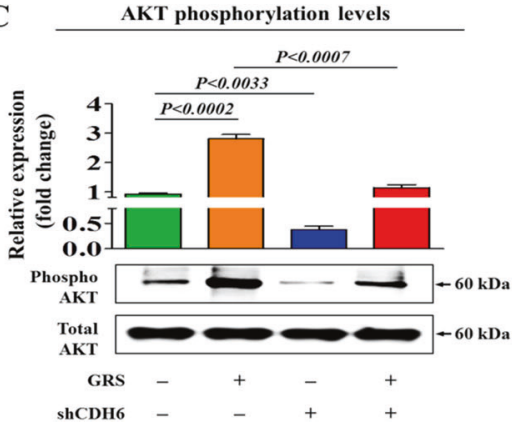

ERK1/2 phosphorylation levels

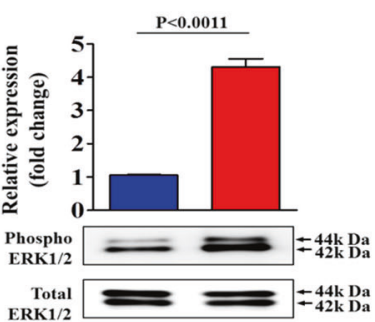

GRS

D

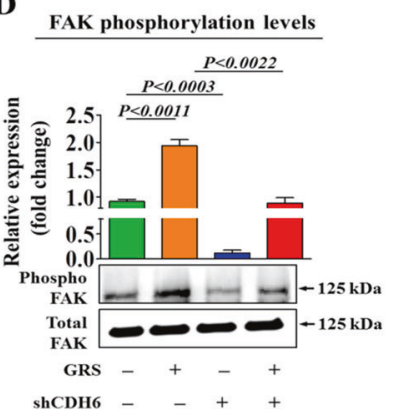

ERK1/2 phosphorylation levels

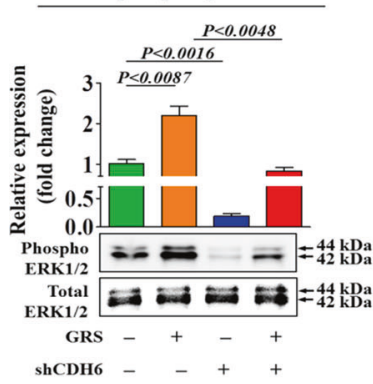

Fig. 4 The stimulatory effects of GRS on PI3K/Akt and/or FAK/ ERK1/2 signaling. MSCs were stimulated for $10 \mathrm{~min}$ with or without GRS $(1 \mu \mathrm{M})$. The cells were then lysed, and the protein contents were analyzed by western blotting using antibodies targeting the phosphorylated forms of PI3K, Akt, CREB FAK, and ERK1/2. The phosphorylation levels of these signaling molecules were significantly

significantly attenuated by treatment with inhibitor $\mathrm{V}$ (Fig. 5a) or PD98059 (Fig. 5b). Consistently, pretreatment with inhibitor V or PD98059 resulted in disruption of the GRS-induced migration (Fig. 5c, d) and MMP-2/9 expression (Fig. 5e, f) of MSCs. The GRS-mediated adipogenic and osteogenic differentiation potential (Fig. 6a, b) and pluripotency-associated factor (NANOG and SOX2) expression (Fig. 6c, d) were also attenuated by treatment with inhibitor V or PD98059. Additionally, to further confirm whether ERK1/2 signaling can regulate GRSmediated functions, we knocked down both ERK1 and 2 expressions using a specific shRNA in MSCs. Successful knockdown of both ERK1 and ERK2 was verified based on RNA and protein levels in MSCs (Supple Fig. 8A-D). Consistently, the GRS-mediated growth (Supple Fig. 9A), migration (Supple Fig. 9B), differentiation potential (Supple increased in cells treated with GRS $(\mathbf{a}, \mathbf{b})$. MSCs were treated with 1 $\mu \mathrm{M}$ GRS alone or were concomitantly transfected with shRNA targeting $\mathrm{CDH}-6$; subsequent changes in the phosphorylation levels of these signaling molecules were measured via western blotting $(\mathbf{c}, \mathbf{d}) . \beta$ actin was used as the internal control. The data represent the mean \pm SD from three independent experiments

Fig. 9C) and pluripotency-associated factor (NANOG and SOX2) expression (Supple Fig. 9D) were also significantly attenuated by both ERK1 and ERK2 knockdown. These results suggest that the PI3K/Akt and/or FAK/ERK1/2 signaling cascade may be involved in multiple beneficial GRSmediated MSC functions, such as growth, differentiation, and migration.

\section{Proteome profiler analysis of GRS-induced secretory protein expression and interconnected signaling networks}

To identify major secreted factors responsible for the beneficial effects of GRS, we analyzed GRS-induced secretory protein expression for multiple common cytokines/growth factors using growth factor antibody arrays. In duplicate 
A
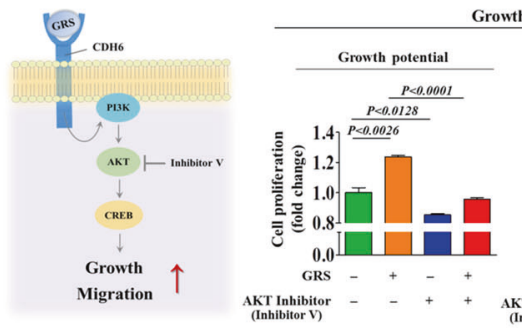

Growth potential

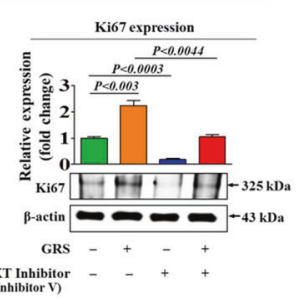

C

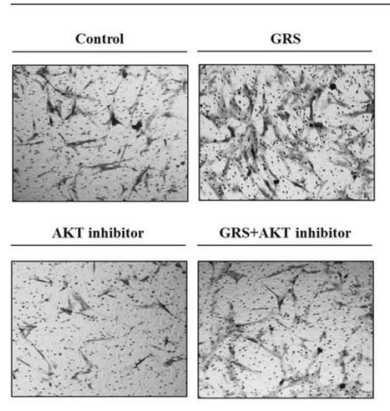

Cell migration

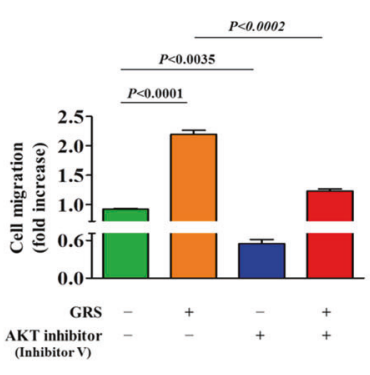

$\mathbf{E}$
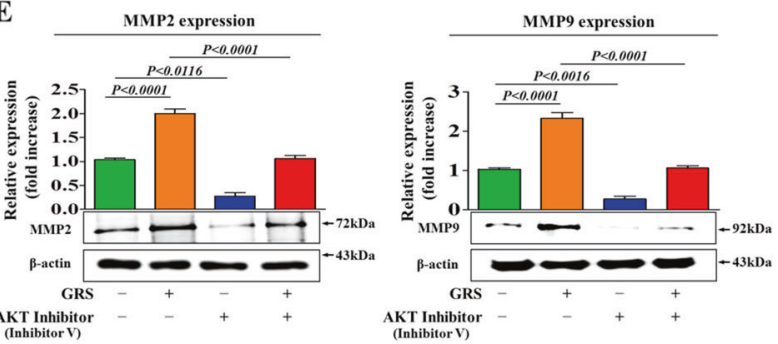

Fig. 5 Inhibition of Akt or ERK1/2 attenuated the GRS-induced proliferative and migratory capacity of MSCs. MSCs were pretreated with inhibitor $\mathrm{V}(10 \mu \mathrm{M})$ or PD98059 $(20 \mu \mathrm{M})$ for $1 \mathrm{~h}$ prior to an additional $48 \mathrm{~h}$ treatment with $1 \mu \mathrm{M}$ GRS; subsequent changes in cell viability were measured by MTT assay and western blotting for proliferation

experiments, we detected 40 proteins in both the stem cell and mock secretomes. The expression levels of six growth factors, namely, glial cell-derived neurotrophic factor (GDNF), macrophage colony-stimulating factor (M-CSF), platelet-derived growth factor (PDGF), stem cell factor $\mathrm{R}$ (SCF R), vascular endothelial growth factor A (VEGF-A), and VEGF R2, were elevated substantially by GRS treatment, whereas the levels of other factors showed only a minor enhancement (Fig. 7a, b). These results suggest that these factors may be at least partly responsible for GRSinduced PI3K/Akt and/or FAK/ERK1/2 signaling and the subsequent beneficial functions. Therefore, we analyzed the activation state of PI3K/Akt or FAK/ERK1/2 signaling and the expression levels of GDNF, M-CSF, PDGF, SCF R, VEGF-A, and VEGF R2 using GeneMANIA (http://www. genemania.org) to evaluate the interconnected signaling networks governing proliferation, differentiation, and migration. To find gene interactions, we considered several factors, including co-expression, co-localization, and
B

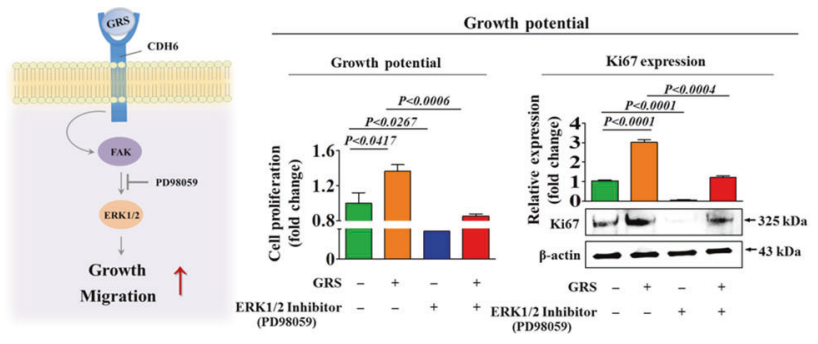

D

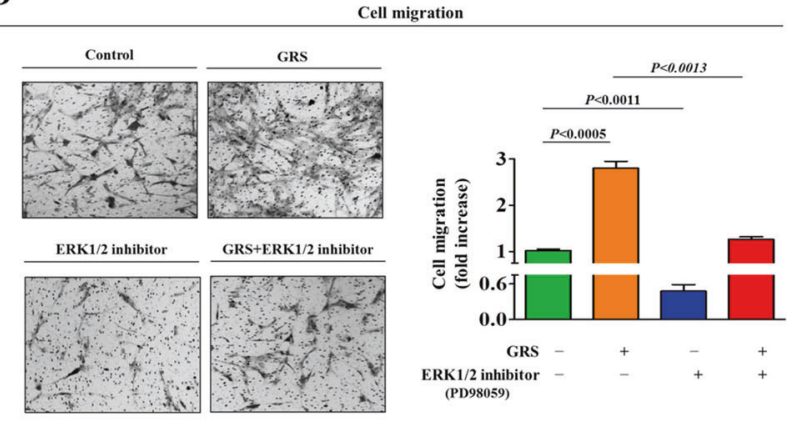

F
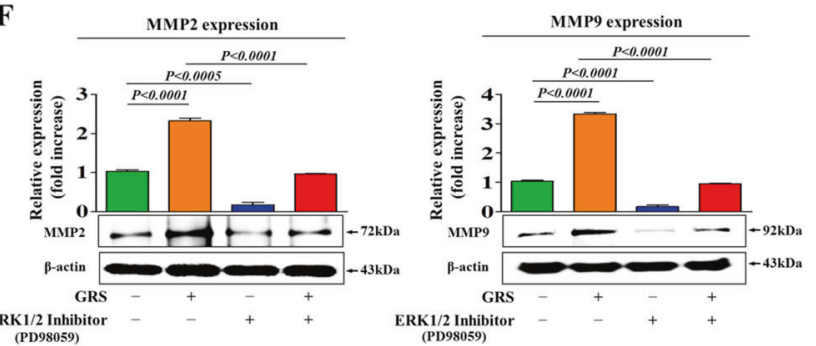

marker Ki67 (a, b). The changes in migratory capacity were measured via the transwell assay (c, d) and western blotting for MMP-2 and MMP-9 (e, f). $\beta$-actin was used as the internal control. The data represent the mean $\pm \mathrm{SD}$ from three independent experiments

genetic interactions. The results revealed a strong relationship between the activation state of PI3K/Akt and/or FAK/ ERK1/2 signaling and the expression levels of the six most prominent factors (Fig. 7c, d).

\section{GRS improves the homing and subsequent therapeutic potential of MSCs in vivo}

Our in vitro data suggested that GRS may act as an injuryinducible danger signal that improves MSC homing potential to the injured site, as well as other beneficial MSC functions. Therefore, we next investigated whether GRS can affect not only the in vivo mobilization of MSCs to sites of injury but also their subsequent therapeutic potential in an animal model of liver fibrosis. To examine the effect of GRS on the regulation of MSC homing to a site of injury, we investigated the in vivo migratory efficacy of MSCs treated with GRS using a green fluorescent protein (GFP) system, which allows the monitoring of transplanted cells. 

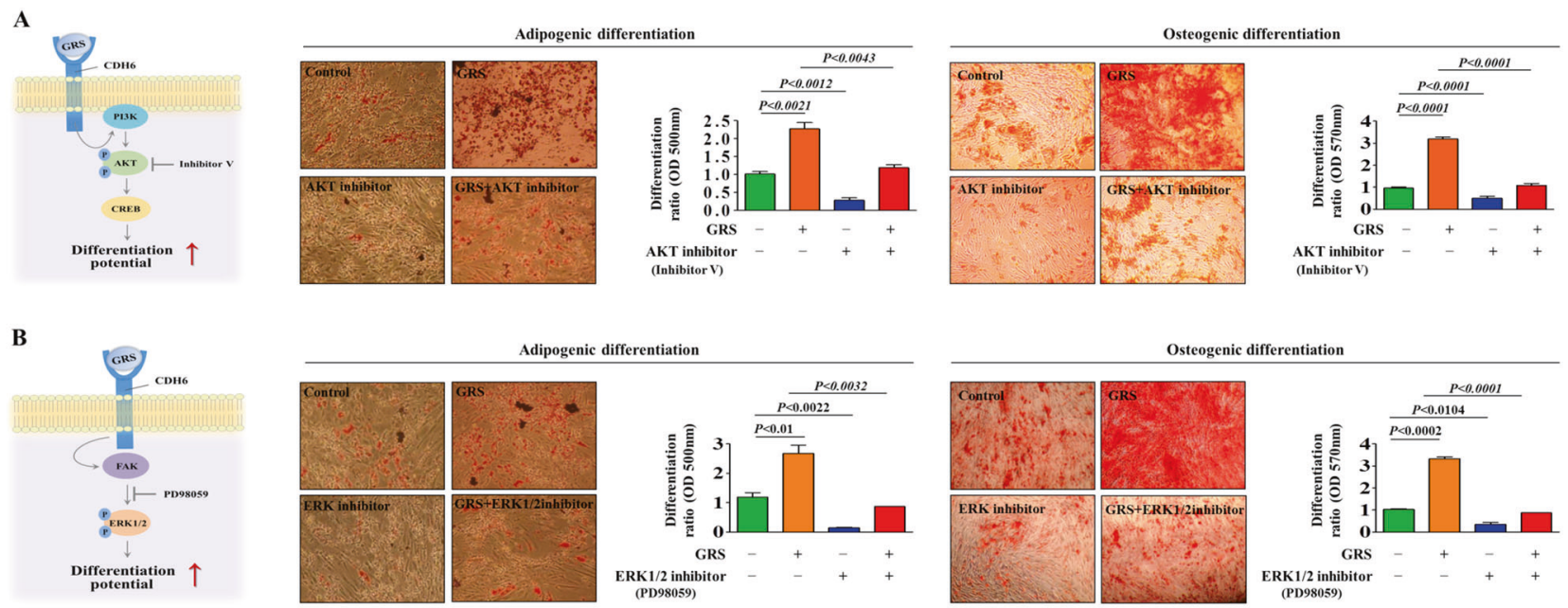
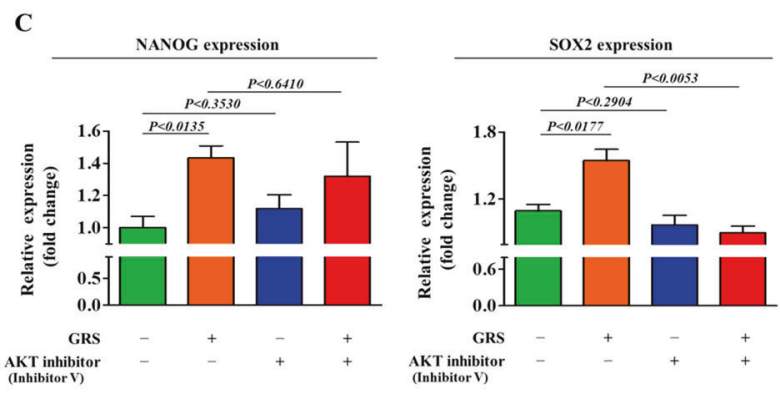

Fig. 6 Inhibition of Akt or ERK1/2 attenuated the GRS-induced differentiation potential of MSCs. MSCs were pretreated with inhibitor V $(10 \mu \mathrm{M})$ or PD98059 $(20 \mu \mathrm{M})$ for $1 \mathrm{~h}$ prior to the additional treatment with $1 \mu \mathrm{M}$ GRS; subsequent changes in osteoblast and adipocyte differentiation were determined by alizarin red and oil red $\mathrm{O}$ staining, respectively. The relative quantification of calcium mineral content and lipid droplet formation was determined by measuring absorbance
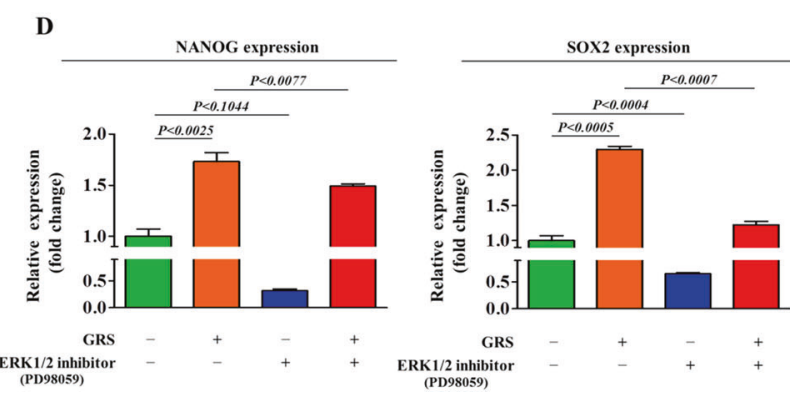

at $570 \mathrm{~nm}$ and $500 \mathrm{~nm}$, respectively (a, b). MSCs were pretreated with inhibitor $\mathrm{V}(10 \mu \mathrm{M})$ or PD98059 $(20 \mu \mathrm{M})$ for $1 \mathrm{~h}$ prior to an additional $24 \mathrm{~h}$ treatment with $1 \mu \mathrm{M}$ GRS; subsequently, the inhibitory effect of GRS-induced stem cell marker expression (SOX2 and NANOG) was determined by real-time PCR $(\mathbf{c}, \mathbf{d})$. The data represent the mean \pm SD from three independent experiments

sites and the subsequent therapeutic potential of MSCs in vivo.

\section{Discussion}

MSCs have been proposed as a potential treatment alternative for various chronic diseases due to their ability to differentiate into mesodermal [26] and ectodermal [27] cells and its marked immunomodulatory capacity in vivo [28]. Despite many promising initial results, some limitations need to be considered. The major challenges of MSC-based therapies are the low abilities of MSCs to differentiate into specialized cell types and migrate to damaged sites [29]. Therefore, several groups have directed their efforts toward finding a novel stimulatory molecule that can improve the differentiation potential and homing efficiency of MSCs. Most recently, particular attention has been devoted to the non-canonical functions of GRS as a novel regulatory cytokine in various human diseases [7-9], as it is selectively 
A

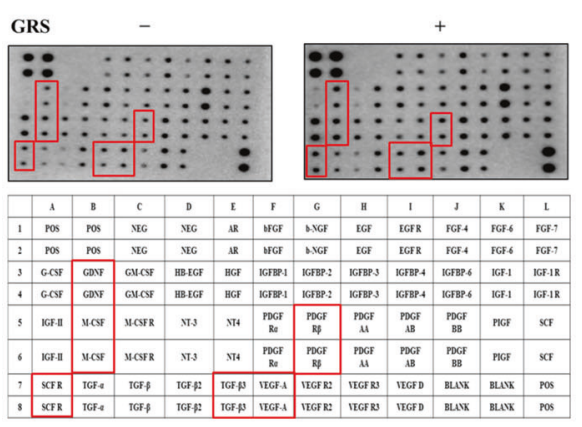

B

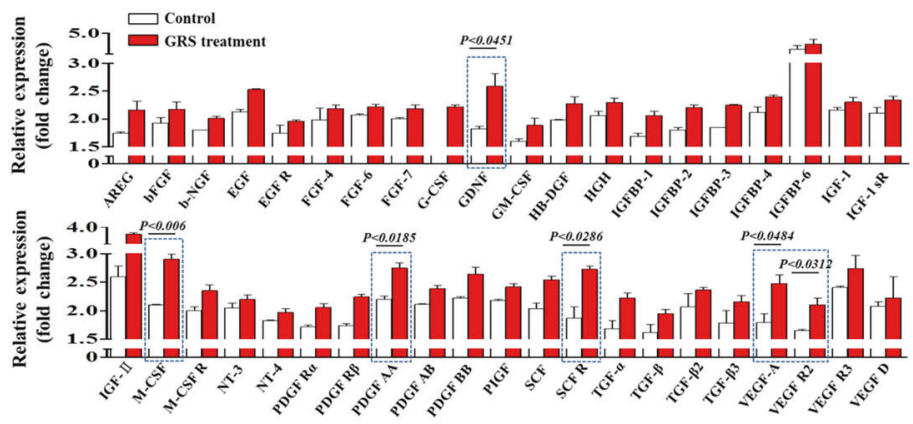

C

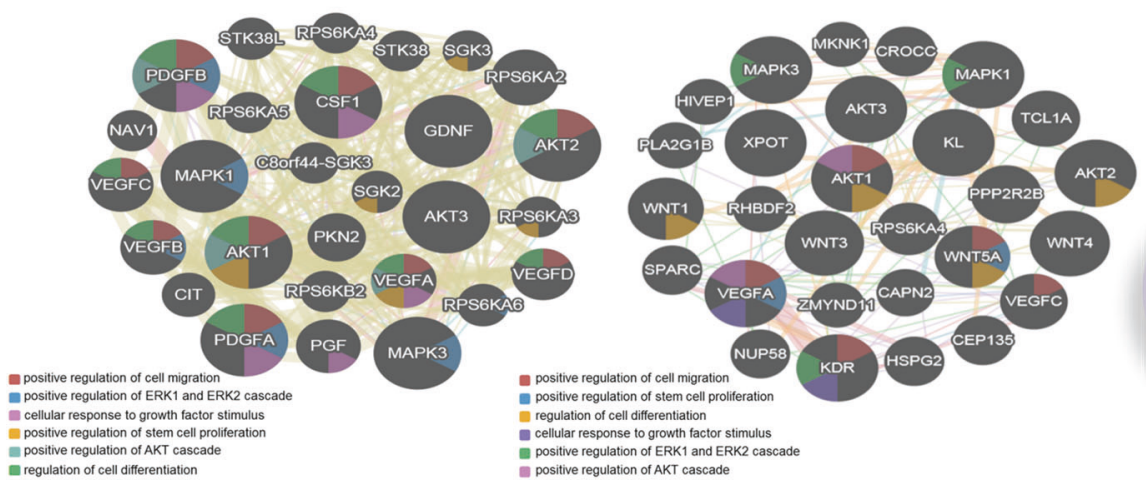

D
Fig. 7 GRS-induced secretory components are associated with PI3K/ Akt and/or FAK/ERK 1/2 signaling. Human growth factor antibody array analysis was performed using GRS-treated and control samples. The membrane was printed with antibodies for 40 growth factors, cytokines, and receptors, with four positive and four negative controls in the upper and lower left corners. Six growth factors or related proteins (GDNF, M-CSF, PDGF, SCF R, VEGF-A, and VEGF R2) were markedly enriched in the GRS-treated groups compared with the control groups $(\mathbf{a}, \mathbf{b})$. Signaling network analysis was performed using GeneMANIA (http://www.genemania.org) to predict the connections between the three growth factors and PI3K/Akt and/or FAK/ERK1/ 2 signaling. The results revealed a positive relationship between each of the six prominent factors (GDNF, M-CSF, PDGF, SCF R, VEGF$\mathrm{A}$, and VEGF R2) and PI3K/Akt and/or FAK/ERK1/2 signaling (c, d). The data represent the mean \pm SD from three independent experiments

differentiation (Fig. 2e, f) in vitro. These results suggest that GRS is actively secreted by multiple cell types in response to injury signals and then acts as a potent stimulatory factor that facilitates multiple beneficial MSCs functions. In addition, GRS-treated MSCs secreted many known growth factors for tissue repair, specifically GDNF, M-CSF, PDGF, and VEGF (Fig. 7). These results indicate that the GRS-mediated therapeutic effect was due at least in part to the secretion of multiple paracrine factors, which regulate many biological processes, including cell migration, apoptosis, growth, and differentiation [33-35].

The exact manner in which the non-canonical functions of GRS were gained has not yet been completely elucidated. Fusing a specialized domain to an existing protein can potentially result in new protein-protein interactions that confer novel functions. Indeed, the expanded functions of the AARS family members are thought to be associated with the acquisition of extra domains [36]. Almost all of the human AARS members have extra domains at either the $\mathrm{N}$ or $\mathrm{C}$ terminus. Although some of these domains are thought 
A
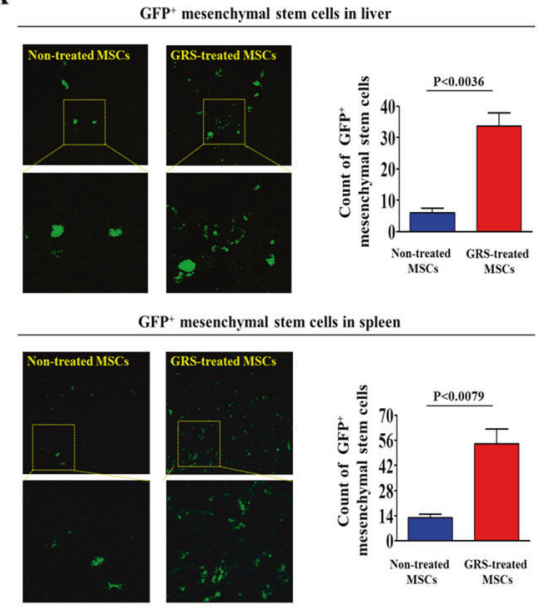

B

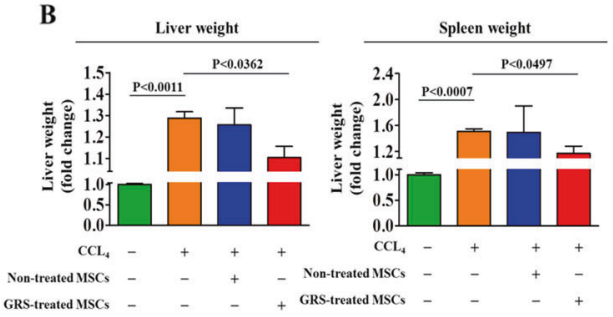

Fig. 8 GRS improves the homing and subsequent therapeutic potential of MSCs in $\mathrm{CCI}_{4}$-induced hepatic fibrosis. Acute $\mathrm{CCl}_{4}(400 \mathrm{mg} / \mathrm{kg})$ treatment (administered intraperitoneally) produced significant histological liver damage compared with the vehicle (corn oil) control. GRS-treated or non-treated MSCs $\left(1 \times 10^{6}\right.$ cells $)$ were labeled with GFP and injected intravenously into the tail veins of 7-week-old NSG mice with acute $\mathrm{CCl}_{4}$-induced liver injury. The mice were sacrificed 7 days after the GFP-labeled MSCs were injected. Green fluorescent images of consecutive sections revealed the presence of GFP-labeled
C
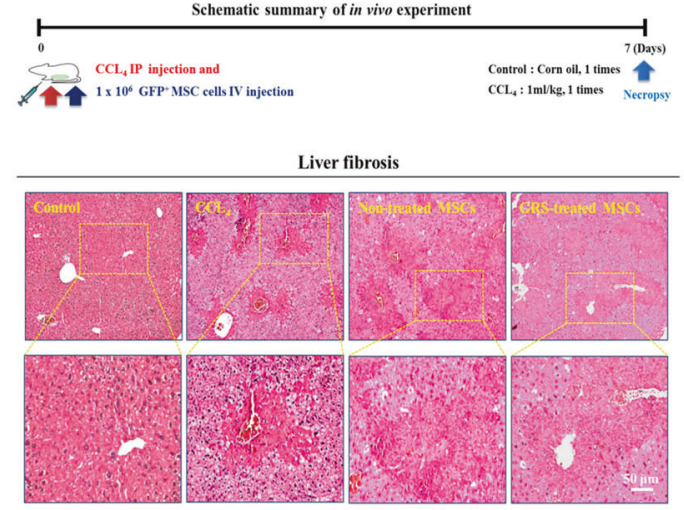

Spleen damage
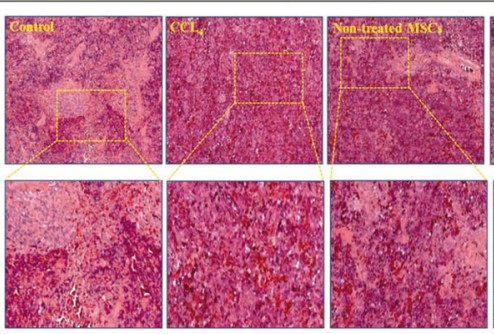

D

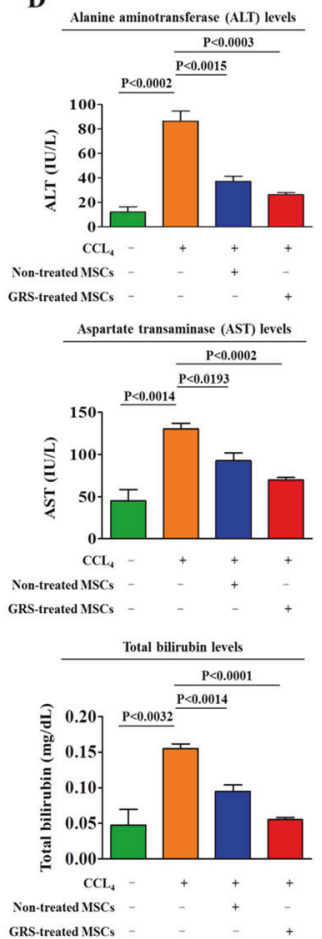

cells (a). The liver and spleen were weighed at the time of sacrifice (b). Liver tissue was collected and subjected to hematoxylin and eosin (H\&E) staining. $\mathrm{CCl}_{4}$-induced liver/spleen fibrosis and centrilobular necrosis were significantly more relieved by the transplantation of GRS-pretreated MSCs (c). Blood samples were obtained from each group 7 days post-MSC transplantation. The serum ALT, AST, and total bilirubin levels were measured using an automatic biochemical analyzer (d). The data represent the mean \pm SD from eight independent experiments

Cell-cell adhesion is mediated by specific interactions between transmembrane cell adhesion molecules (CAMs), such as cadherins, integrins, and selectins [38]. Because members of the CAM family of proteins are involved in regulating multiple aspects of cellular behavior, including cell migration, growth, apoptosis, and differentiation [39], the role of GRS in activating CDH-6 implies its potential role in the maintenance of stem cell self-renewal and differentiation. Because the cadherin family is known to activate the Rho/Rac signaling pathways for the regulation of adhesion dynamics [40,41], we also investigated whether GRS activates the Rho/Rac downstream effectors Akt and ERK1/2 when GRS binds to CDH-6 in MSCs. Generally, the PI3K/Akt [42] and FAK/ERK1/2 [43] signaling pathways are preferentially activated by mitogens and growth factors in multiple cell types. Our data show that GRS activated PI3K/Akt and/or FAK/ERK1/2 signaling in MSCs (Fig. 4a, b). Indeed, GRS-induced PI3K/Akt and/or FAK/ ERK1/2 signaling was significantly attenuated by K-cadherin depletion (Fig. 4c, d). The role of GRS in regulating these signaling pathways is not surprising, 
considering the previous in vitro studies on ECs, which revealed that truncated tryptophanyl tRNA synthetase (TrpRS) significantly suppresses stress-induced cellular responses involved in angiogenesis, such as EC migration and proliferation [4, 44] and Akt and ERK1/2 signaling pathway activation [45]. Blockage of these signaling pathways with specific inhibitors resulted in significant attenuation of the GRS-mediated proliferation (Fig. 5a, b), migration (Fig. 5c-f), and differentiation (Fig. 6) of the responding cells, suggesting that GRS exerts its biological activities on responding cells via PI3K/Akt and/or FAK/ ERK1/2 signaling

Taken together, these findings suggest that in addition to its previously reported canonical activities, GRS is actively secreted in response to tissue damage as an endogenous danger signal and subsequently enhances the therapeutic effects of MSCs by promoting their differentiation and migration through non-canonical PI3K/Akt and/or FAK/ ERK1/2 signaling. This study also provides novel insights into the molecular mechanisms regulating the differentiation and migration potential of MSCs with relevance to clinical applications.

\section{Materials and methods}

\section{Isolation and culture of human adipose tissue- derived mesenchymal stem cells}

Human adipose tissue-derived mesenchymal stem cells were obtained from adipose tissues of breast cancer patients with written informed consent from the patients and approval of the Gachon University Institutional Review Board (IRB No: GAIRB2015-104). All of the humanrelated experiments were approved and conducted in accordance with the Gachon University Institutional Review Board (IRB No: GAIRB2015-104). Adipose tissue was minced into small pieces, and then the small pieces were digested in DMEM containing 10\% FBS and $250 \mathrm{U} /$ $\mathrm{ml}$ type I collagenase for $5 \mathrm{~h}$ at $37^{\circ} \mathrm{C}$. The digestion mixture was then filtered through a $40 \mu \mathrm{m}$ cell strainer. Isolated cells were then cultured in EBM-2 medium (Lonza) with EGM-2 supplements at $37^{\circ} \mathrm{C}$ and $5 \% \mathrm{CO}_{2}$.

\section{In vitro cell migration assay}

Cells were plated at $1 \times 10^{5}$ cells/well in $200 \mu \mathrm{L}$ of culture medium in the upper chambers of transwell permeable supports (Corning Inc., Corning, NY, USA) to track the migration of cells. The transwell chambers had $8.0 \mu \mathrm{m}$ pores in $6.5-\mathrm{mm}$ diameter polycarbonate membranes and used a 24-well plate format. Non-invading cells on the upper surface of each membrane were removed by scrubbing with laboratory paper. Migrated cells on the lower surface of each membrane were fixed with $4 \%$ paraformaldehyde for $5 \mathrm{~min}$ and stained with hematoxylin for $15 \mathrm{~min}$. Later, the number of migrated cells was counted in three randomly selected fields of the wells under a light microscope at $\times 50$ magnification. To calculate the chemotactic index, the number of cells that migrated in response to the treatment of GRS was divided by the number of spontaneously migrating cells.

\section{Cell proliferation assay}

The MTT assay was used to determine the proliferative capacity of GRS, according to the manufacturer's protocol. Cells $\left(1 \times 10^{4}\right.$ cells/well $)$ were seeded in 96-well plates. After $24 \mathrm{~h}$ of incubation, the cells were treated with GRS or vehicle for $72 \mathrm{~h}$. The viable cells were measured at $570 \mathrm{~nm}$ using a VersaMax microplate reader.

\section{Osteogenic differentiation}

MSCs were incubated in DMEM high-glucose medium supplemented with $0.1 \mu \mathrm{M}$ dexamethasone, $10 \mathrm{mM} \beta$-glycerophosphate, $50 \mu \mathrm{M}$ ascorbate and $10 \%$ FBS with or without dioxin. MSCs were grown for 3 weeks, with medium replacement twice a week. Differentiated cells were stained with Alizarin Red $\mathrm{S}$ to detect de novo formation of bone matrix. Alizarin red $\mathrm{S}$ in samples was quantified by measuring the optical density (OD) of the solution at $570 \mathrm{~nm}$.

\section{Adipogenic differentiation}

MSCs were incubated in DMEM low-glucose medium supplemented with $500 \mu \mathrm{M}$ metylxanthine, $5 \mu \mathrm{g} / \mathrm{mL}$ insulin, and $10 \%$ FBS with or without dioxin. MSCs were grown for 3 weeks, with medium replacement twice a week. Lipid droplet formation was confirmed by Oil Red O staining. Relative quantification of lipid droplet formation was determined by absorbance measurement at $500 \mathrm{~nm}$.

\section{Immunofluorescent staining}

Samples were fixed with $4 \%$ paraformaldehyde for fluorescent staining. Samples were permeabilized with $0.4 \mathrm{M}$ glycine and $0.3 \%$ Triton $\mathrm{X}-100$, and nonspecific binding was blocked with $2 \%$ normal swine serum (DAKO, Glostrup, Denmark). Staining was performed as described previously [46], using the primary anti-Phalloidin (Cytoskeleton, Inc.) antibody. Samples were examined by fluorescence microscopy (Zeiss LSM 510 Meta). The calculation of expression was based on green fluorescence area and density divided by cell number, as determined from the 
number of DAPI-stained nuclei, in three randomly selected fields for each sample from a total of three independent experiments.

\section{$\mathrm{CCl}_{4}$-induced acute liver failure}

All of the animal experiments were approved and conducted in accordance with the Institutional Animal Care and Use Committee (IACUC) (LCDI-2016-0083) of the Lee Gil Ya Cancer and Diabetes Institute of Gachon University. 7week-old NSG mice were subjected to treatment with $\mathrm{CCl}_{4}$ to induce liver injury, or with sterilized corn oil vehicle as control. The animals were divided into two experimental groups ( $n=8$ mice for each group): (a) mice treated with $\mathrm{CCl}_{4}$; (b) mice treated with corn oil. $\mathrm{CCl}_{4}(400 \mathrm{mg}$ per $\mathrm{kg}$ of animal, in a $10 \%$ solution of corn oil) or corn oil were injected intraperitoneally. Mice of each group were sacrificed by cervical dislocation. Before sacrifice, the mice were anesthetized and exsanguinated by cardiac puncture to obtain blood serum. Blood samples were obtained from each group on days 7 post-MSC transplantation. The samples were immediately centrifuged at $2000 \times g$ for $10 \mathrm{~min}$ before serum was collected. The ALT, AST, and total bilirubin levels were measured using a serum multiple automatic biochemical analyzer. Livers and spleen were washed with ice-cold PBS and dissected into smaller pieces. Liver sections were deparaffinized, rehydrated, and stained with hematoxylin and eosin (H\&E).

\section{MSC labeling and tracking in mouse model}

MSCs were trypsin released, centrifuged and re-suspended at a concentration of $2 \times 10^{5}$ cells $/ \mathrm{ml}$. Subsequently, MSCs were transfected with GFP-expressing plasmid vectors according to the manufacturer's instructions. Their expression efficiency was analyzed using an immunocytochemistry after $48 \mathrm{~h}$ of transfection. Immediately after labeling MSCs were collected, centrifuged and re-suspended in $40 \mu \mathrm{l}$ growth media which were subsequently injected intravenously (i.v.) via the lateral tail vein of mice after acute liver injury with $\mathrm{CCl}_{4}$. Following injection of GFP-labeled MSCs, mice were sacrificed 7 days after injection and subsequently the distribution of MSCs were compared between the control and $\mathrm{CCl}_{4}$-injected groups under fluorescence microscope.

\section{Flow cytometry}

FACS analysis and cell sorting were performed using FACS Calibur and FACS Aria machines (Becton Dickinson, Palo Alto, CA), respectively. FACS data were analyzed using FlowJo software (Tree Star, Ashland, OR). Antibodies to the following proteins were used: APC-conjugated CD44
(BD Bioscience, Cat. 559942, dilution 1/40), PE-conjugated CD133 (MACS; Miltenyi Biotech, Sunnyvale, CA, 130080-081, dilution 1/40), CD34 (MACS; Miltenyi Biotech, Sunnyvale, CA, 30-081-002), CD44 (MACS; Miltenyi Biotech, Sunnyvale, CA, 130-095-180), CD45 (MACS; Miltenyi Biotech, Sunnyvale, CA, 130-080-201), CD73 (MACS; Miltenyi Biotech, Sunnyvale, CA, 130-095-182) and CD105 (MACS; Miltenyi Biotech, Sunnyvale, CA, 130-094-941) The FACS gates were established by staining with an isotype antibody or secondary antibody.

\section{Protein isolation and western blot analysis}

The protein expression levels were determined by western blot analysis as previously described [47]. Cells were lysed in a buffer containing $50 \mathrm{mM}$ Tris, $5 \mathrm{mM}$ EDTA, $150 \mathrm{mM}$ $\mathrm{NaCl}, 1 \mathrm{mM}$ DTT, $0.01 \% \mathrm{NP} 40$, and $0.2 \mathrm{mM}$ PMSF. The protein concentrations of the total cell lysates were measured by using bovine serum albumin as a standard. Samples containing equal amounts of protein were separated via sodium dodecyl sulfate-polyacrylamide gel electrophoresis (SDS-PAGE) and then transferred onto nitrocellulose membranes (Bio-Rad Laboratories). The membranes were blocked with $5 \%$ skim milk in Tris-buffered saline containing Tween-20 at RT. Then, the membranes were incubated with primary antibodies against $\beta$-actin (Abcam, MA, USA, ab189073), MMP-2 (Cell signaling \#4022), MMP-9 (Cell Signaling \#13667), total PI3K (Cell Signaling \#4292), phospho-PI3K (Cell Signaling \#4228), total CREB (Cell Signaling \#9197), phospho-CREB (Cell Signaling \#9198), total Akt (Cell Signaling \#4491), phospho-Akt (Cell Signaling \#4060), total-ERK1/2 (Cell Signaling \#9012), phospho-ERK1/2 (Cell Signaling \#9101), Ki67 (Novus, NB500-170), total FAK (Santa Cruz, sc-558), and phosphoFAK (Santa Cruz, sc-11765) overnight at $4{ }^{\circ} \mathrm{C}$ and then with HRP-conjugated goat anti-rabbit IgG (BD Pharmingen, San Diego, CA, USA, 554021) and goat anti-mouse IgG (BD Pharmingen, 554002) secondary antibodies for 60 min at RT. Antibody-bound proteins were detected using ECL reagents.

\section{Real-time PCR}

Total RNA from skin cells was extracted using TRIzol reagent (Invitrogen) according to the manufacturer's protocol. The first-strand cDNA was synthesized with 1 to $2 \mu \mathrm{g}$ of total RNA using SuperScript II (Invitrogen), and onetenth of the cDNA was used for each PCR mixture containing Express SYBR-Green qPCR Supermix (BioPrince, Seoul, South Korea). Real-time PCR was performed using a Rotor-Gene Q (Qiagen). The reaction was subjected to amplification cycles of $95^{\circ} \mathrm{C}$ for $20 \mathrm{~s}, 60^{\circ} \mathrm{C}$ for $20 \mathrm{~s}$ and 72 ${ }^{\circ} \mathrm{C}$ for $25 \mathrm{~s}$. The relative mRNA expression of the selected 
genes was normalized to that of PPIA and quantified using the $\Delta \Delta \mathrm{CT}$ method. The sequences of the PCR primers are listed in supplementary table 1.

\section{GeneMANIA algorithm-based bioinformatics analysis}

To further analyze genes that interact with or directly regulate PI3K/Akt or FAK/ERK1/2 signaling, we imported all identified genes and their corresponding accession numbers into GeneMANIA (http://www.genemania.org). To find gene interactions, we considered several factors including co-expression, co-localization, and genetic interactions. From this list, we selected the genes GDNF, M-CSF, PDGF, SCF R, VEGF-A, and VEGF R2 to test their involvement in regulating GRS-induced PI3K/Akt or FAK/ ERK1/2 signaling.

\section{Growth factor antibody array}

The assay was performed following the manufacturer's protocol (Abnova AA0089). Briefly, GRS or vehicle-treated protein samples were incubated with antibody membranes overnight at $4{ }^{\circ} \mathrm{C}$. After washing three times with wash buffer, the membranes were incubated with biotinconjugated anti-cytokine antibodies overnight at $4{ }^{\circ} \mathrm{C}$. The membranes were then washed three times and incubated with HRP-conjugated streptavidin. Chemiluminescence was used to detect signals of the growth factors spotted on the nitrocellulose membrane.

\section{Statistical analysis}

All the statistical data were analyzed in GraphPad Prism 5.0 (GraphPad Software, San Diego, CA) and evaluated using two-tailed Student's $t$-tests. Values of $P<0.05$ were considered to indicate statistical significance.

Acknowledgements This research was supported by Basic Science Research Program through the National Research Foundation of Korea (NRF) funded by the Ministry of Science, ICT and Future Planning (NRF-2016R1C1B2009351 and NRF-2015R1C1A1A02037004).

\section{Compliance with ethical standards}

Conflict of interest The authors declare that they have no conflict of interest.

\section{References}

1. Ibba M, Soll D. Aminoacyl-tRNA synthesis. Annu Rev Biochem. 2000;69:617-50.

2. Park SG, Kang YS, Kim JY, Lee CS, Ko YG, Lee WJ, et al. Hormonal activity of AIMP1/p43 for glucose homeostasis. Proc Natl Acad Sci USA. 2006;103:14913-8.
3. Ko YG, Park H, Kim T, Lee JW, Park SG, Seol W, et al. A cofactor of tRNA synthetase, p43, is secreted to up-regulate proinflammatory genes. J Biol Chem. 2001;276:23028-33.

4. Wakasugi K, Slike BM, Hood J, Otani A, Ewalt KL, Friedlander $\mathrm{M}$, et al. A human aminoacyl-tRNA synthetase as a regulator of angiogenesis. Proc Natl Acad Sci USA. 2002;99:173-7.

5. Park SG, Shin H, Shin YK, Lee Y, Choi EC, Park BJ, et al. The novel cytokine p43 stimulates dermal fibroblast proliferation and wound repair. Am J Pathol. 2005;166:387-98.

6. Han JM, Myung H, Kim S. Antitumor activity and pharmacokinetic properties of ARS-interacting multi-functional protein 1 (AIMP1/p43). Cancer Lett. 2010;287:157-64.

7. Antonellis A, Ellsworth RE, Sambuughin N, Puls I, Abel A, LeeLin SQ, et al. Glycyl tRNA synthetase mutations in CharcotMarie-Tooth disease type 2D and distal spinal muscular atrophy type V. Am J Hum Genet. 2003;72:1293-9.

8. Xie W, Schimmel P, Yang XL. Crystallization and preliminary Xray analysis of a native human tRNA synthetase whose allelic variants are associated with Charcot-Marie-Tooth disease. Acta Crystallogr Sect F Struct Biol Cryst Commun. 2006;62:1243-6.

9. Cader MZ, Ren J, James PA, Bird LE, Talbot K, Stammers DK. Crystal structure of human wildtype and S581L-mutant glycyltRNA synthetase, an enzyme underlying distal spinal muscular atrophy. FEBS Lett. 2007;581:2959-64.

10. Caplan AI. Mesenchymal stem cells. J Orthop Res. 1991;9:641-50.

11. Gong W, Han Z, Zhao H, Wang Y, Wang J, Zhong J, et al. Banking human umbilical cord-derived mesenchymal stromal cells for clinical use. Cell Transplant. 2012;21:207-16.

12. Yellowley C. CXCL12/CXCR4 signaling and other recruitment and homing pathways in fracture repair. Bone Rep. 2013;2:300.

13. Honczarenko M, Le Y, Swierkowski M, Ghiran I, Glodek AM, Silberstein LE. Human bone marrow stromal cells express a distinct set of biologically functional chemokine receptors. Stem Cells. 2006;24:1030-41.

14. Ringe J, Strassburg S, Neumann K, Endres M, Notter M, Burmester GR, et al. Towards in situ tissue repair: human mesenchymal stem cells express chemokine receptors CXCR1, CXCR2 and CCR2, and migrate upon stimulation with CXCL8 but not CCL2. J Cell Biochem. 2007;101:135-46.

15. Forte G, Minieri M, Cossa P, Antenucci D, Sala M, Gnocchi V, et al. Hepatocyte growth factor effects on mesenchymal stem cells: proliferation, migration, and differentiation. Stem Cells. 2006;24:23-33.

16. Gharibi B, Ghuman MS, Hughes FJ. Akt- and Erk-mediated regulation of proliferation and differentiation during PDGFRbetainduced MSC self-renewal. J Cell Mol Med. 2012;16:2789-801.

17. Zheng B, Wang C, He L, Xu X, Qu J, Hu J, et al. Neural differentiation of mesenchymal stem cells influences chemotactic responses to HGF. J Cell Physiol. 2013;228:149-62.

18. Song BQ, Chi Y, Li X, Du WJ, Han ZB, Tian JJ, et al. Inhibition of notch signaling promotes the adipogenic differentiation of mesenchymal stem cells through autophagy activation and PTEN$\mathrm{PI} 3 \mathrm{~K} / \mathrm{AKT} / \mathrm{mTOR}$ pathway. Cell Physiol Biochem. 2015;36:1991-2002.

19. Tang JM, Yuan J, Li Q, Wang JN, Kong X, Zheng F, et al. Acetylcholine induces mesenchymal stem cell migration via $\mathrm{Ca} 2$ $+/$ PKC/ERK1/2 signal pathway. J Cell Biochem. 2012;113:2704-13.

20. Park MC, Kang T, Jin D, Han JM, Kim SB, Park YJ, et al. Secreted human glycyl-tRNA synthetase implicated in defense against ERK-activated tumorigenesis. Proc Natl Acad Sci USA. 2012;109:E640-647.

21. Yamaguchi H, Condeelis J. Regulation of the actin cytoskeleton in cancer cell migration and invasion. Biochim Biophys Acta. 2007; 1773:642-52. 
22. Zhou Q, Kapoor M, Guo M, Belani R, Xu X, Kiosses WB, et al. Orthogonal use of a human tRNA synthetase active site to achieve multifunctionality. Nat Struct Mol Biol. 2010;17:57-61.

23. Armstrong L, Hughes O, Yung S, Hyslop L, Stewart R, Wappler I, et al. The role of PI3K/AKT, MAPK/ERK and NFkappabeta signalling in the maintenance of human embryonic stem cell pluripotency and viability highlighted by transcriptional profiling and functional analysis. Hum Mol Genet. 2006;15:1894-913.

24. Muller P, Langenbach A, Kaminski A, Rychly J. Modulating the actin cytoskeleton affects mechanically induced signal transduction and differentiation in mesenchymal stem cells. PLoS ONE. 2013;8:e71283.

25. Gao F, Hu X, Xie X, Liu X, Wang J. Heat shock protein 90 stimulates rat mesenchymal stem cell migration via PI3K/Akt and ERK1/2 pathways. Cell Biochem Biophys. 2015;71:481-9.

26. Dezawa M, Ishikawa $H$, Itokazu $Y$, Yoshihara $T$, Hoshino M, Takeda $\mathrm{S}$, et al. Bone marrow stromal cells generate muscle cells and repair muscle degeneration. Science. 2005;309:314-7.

27. Dezawa M, Takahashi I, Esaki M, Takano M, Sawada H. Sciatic nerve regeneration in rats induced by transplantation of in vitro differentiated bone-marrow stromal cells. Eur J Neurosci. 2001;14:1771-6.

28. Abdi R, Fiorina P, Adra CN, Atkinson M, Sayegh MH. Immunomodulation by mesenchymal stem cells: a potential therapeutic strategy for type 1 diabetes. Diabetes. 2008;57:1759-67.

29. Mezey E, Chandross KJ, Harta G, Maki RA, McKercher SR. Turning blood into brain: cells bearing neuronal antigens generated in vivo from bone marrow. Science. 2000;290:1779-82.

30. Scandurro AB, Weldon CW, Figueroa YG, Alam J, Beckman BS. Gene microarray analysis reveals a novel hypoxia signal transduction pathway in human hepatocellular carcinoma cells. Int $\mathbf{J}$ Oncol. 2001;19:129-35.

31. Motley WW, Seburn KL, Nawaz MH, Miers KE, Cheng J, Antonellis A, et al. Charcot-Marie-Tooth-linked mutant GARS is toxic to peripheral neurons independent of wild-type GARS levels. PLoS Genet. 2011;7:e1002399.

32. Howard OM, Dong HF, Yang D, Raben N, Nagaraju K, Rosen A, et al. Histidyl-tRNA synthetase and asparaginyl-tRNA synthetase, autoantigens in myositis, activate chemokine receptors on $\mathrm{T}$ lymphocytes and immature dendritic cells. J Exp Med. 2002;196:781-91.

33. Hocking AM, Gibran NS. Mesenchymal stem cells: paracrine signaling and differentiation during cutaneous wound repair. Exp Cell Res. 2010;316:2213-9.

34. Smith AN, Willis E, Chan VT, Muffley LA, Isik FF, Gibran NS, et al. Mesenchymal stem cells induce dermal fibroblast responses to injury. Exp Cell Res. 2010;316:48-54.
35. Lee EY, Xia Y, Kim WS, Kim MH, Kim TH, Kim KJ, et al. Hypoxia-enhanced wound-healing function of adipose-derived stem cells: increase in stem cell proliferation and up-regulation of VEGF and bFGF. Wound Repair Regen. 2009;17:540-7.

36. Guo M, Schimmel P, Yang XL. Functional expansion of human tRNA synthetases achieved by structural inventions. FEBS Lett. 2010;584:434-42.

37. Wakasugi K, Schimmel P. Two distinct cytokines released from a human aminoacyl-tRNA synthetase. Science. 1999;284:147-51.

38. Aplin AE, Howe A, Alahari SK, Juliano RL. Signal transduction and signal modulation by cell adhesion receptors: the role of integrins, cadherins, immunoglobulin-cell adhesion molecules, and selectins. Pharmacol Rev. 1998;50:197-263.

39. Okegawa T, Pong RC, Li Y, Hsieh JT. The role of cell adhesion molecule in cancer progression and its application in cancer therapy. Acta Biochim Pol. 2004;51:445-57.

40. Nakagawa M, Fukata M, Yamaga M, Itoh N, Kaibuchi K. Recruitment and activation of Racl by the formation of Ecadherin-mediated cell-cell adhesion sites. J Cell Sci. 2001;114:1829-38.

41. Kovacs EM, Goodwin M, Ali RG, Paterson AD, Yap AS. Cadherin-directed actin assembly: E-cadherin physically associates with the Arp2/3 complex to direct actin assembly in nascent adhesive contacts. Curr Biol. 2002;12:379-82.

42. Chen J, Crawford R, Chen C, Xiao Y. The key regulatory roles of the PI3K/Akt signaling pathway in the functionalities of mesenchymal stem cells and applications in tissue regeneration. Tissue Eng Part B Rev. 2013;19:516-28.

43. Huang C, Jacobson K, Schaller MD. MAP kinases and cell migration. J Cell Sci. 2004;117:4619-28.

44. Otani A, Slike BM, Dorrell MI, Hood J, Kinder K, Ewalt KL, et al. A fragment of human TrpRS as a potent antagonist of ocular angiogenesis. Proc Natl Acad Sci USA. 2002;99:178-83.

45. Tzima E, Reader JS, Irani-Tehrani M, Ewalt KL, Schwartz MA, Schimmel P. Biologically active fragment of a human tRNA synthetase inhibits fluid shear stress-activated responses of endothelial cells. Proc Natl Acad Sci USA. 2003;100:14903-7.

46. Dong HJ, Jang GB, Lee HY, Park SR, Kim JY, Nam JS, et al. The Wnt/beta-catenin signaling/Id2 cascade mediates the effects of hypoxia on the hierarchy of colorectal-cancer stem cells. Sci Rep. 2016;6:22966.

47. Choi ES, Jung JY, Lee JS, Park JH, Cho NP, Cho SD. Myeloid cell leukemia-1 is a key molecular target for mithramycin Ainduced apoptosis in androgen-independent prostate cancer cells and a tumor xenograft animal model. Cancer Lett. 2013;328:65-72. 